\title{
Erratum to: Validation of a new meteorological forcing data in analysis of spatial and temporal variability of precipitation in India
}

Lu Li $\cdot$ Chong-Yu Xu $\cdot$ Zengxin Zhang

Sharad K. Jain

Published online: 4 March 2014

(C) Springer-Verlag Berlin Heidelberg 2014

Erratum to: Stoch Environ Res Risk Assess (2014)

28:239-252

DOI 10.1007/s00477-013-0745-7

The figure caption for Fig. 1 is updated. Please find here the figure with updated caption.

The online version of the original article can be found under doi:10.1007/s00477-013-0745-7.

\section{Li}

Uni Climate, Uni Research, Bergen, Norway

e-mail: marylilu420@gmail.com

L. Li

Bjerknes Centre for Climate Research, Bergen, Norway

L. Li

Department of Geosciences, University of Oslo, Postboks 7801,

5020 Bergen, Norway

\section{C.-Y. Xu ( $₫)$}

Department of Geosciences, University of Oslo, P.O. Box 1047, Blindern, 0316 Oslo, Norway

e-mail: c.y.xu@geo.uio.no

\section{C.-Y. Xu}

Department of Earth Sciences, Uppsala University, Uppsala, Sweden

\section{Z. Zhang}

Jiangsu Key Laboratory of Forestry Ecological Engineering,

Nanjing Forestry University, Nanjing 210037, China

S. K. Jain

National Institute of Hydrology, Roorkee 247 667, India

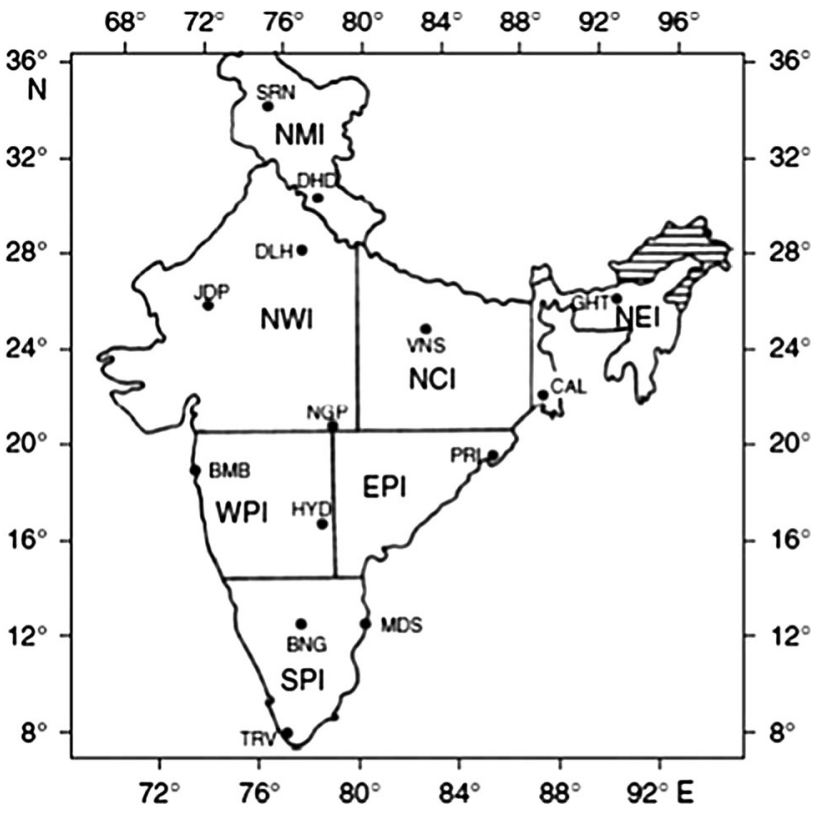

Fig. 1 The boundary of 7 homogeneous rainfall zones (from Sontakke et al. 2008) 